Fecha de recepción: septiembre 2019 Fecha de aceptación: noviembre 2019 Versión final: febrero 2020

\section{Universidad y trabajo: alianza 5.0. Perspectivas y devenires desde el aula}

Débora Irina Belmes ${ }^{(1)}$

\begin{abstract}
Resumen: La propuesta de este trabajo es compartir algunas reflexiones sobre el lugar que el conocimiento universitario ocupa en su vinculación con el contexto socio-cultural y el mundo del trabajo. El siglo XXI se presenta como un escenario cambiante y convulsionado por las nuevas formas del capitalismo, los cambios en las comunicaciones, la tecnologización de la vida cotidiana, la creciente centralización de lo urbano, las modificaciones en el consumo, y las reconversiones en el mundo laboral que han transformado los requerimientos para los puestos de trabajo. La pregunta por la formación no es ingenua. La educación en la Argentina históricamente ha tenido un lugar importante, que ha generado debates y tensiones. En el plano del campo universitario el momento actual plantea una serie de desafíos no sólo en cuanto a sus contenidos sino también en lo referido los modos en que se implementan sus prácticas. Qué hay que enseñar, qué se puede pensar acerca de la transmisión de conocimientos, quiénes son los docentes y quiénes los alumnos, cuáles son las propuestas acerca de la transformación del aula como un espacio de encuentro y cuál es la relación con el futuro, son algunas de las preguntas que guían la presente ponencia.
\end{abstract}

Palabras clave: formación universitaria - requerimientos laborales - aula - transmisión docentes - alumnos.

[Resúmenes en inglés y portugués en las páginas 56-57]

(1) Lic. En Sociología y Psicología (UBA). Profesora en Enseñanza Normal y Especial en Sociología (UBA) Profesora en la Facultad de Diseño y Comunicación de la Universidad de Palermo. Miembro del Consejo Asesor Académico de esa Facultad.

Las relaciones entre las universidades y el mundo del trabajo siempre han sido dinámicas y múltiples pero el paso del siglo ha dejado sus marcas. Las universidades se han transformado, su número es mayor y la población que la habita y sostiene también se ha incrementado de manera notable. Su lugar en la sociedad presenta nuevos desafíos ya que podría señalarse en líneas generales que su existencia está relacionada con la posibilidad de generar y resolver cuestiones vinculadas al conocimiento y en proveer mano profesional al mundo de la producción. Desde esta mirada se propone que es el incremento de la 
educación a través no solo del grado sino de los posgrados y especializaciones constituye una de las vías para sostener el ritmo que plantea la carrera entre la educación y el cambio tecnológico. Una de las dificultades que esta carrera bosqueja es el tema de la velocidad ya que los programas educativos plantean tiempos muy distintos a los del ritmo tecnológico tanto en su ejecución como en su evaluación. Sin embargo, la presión es incesante y las instituciones educativas deben actualizar y promover nuevas áreas para que sus profesionales tengan las competencias necesarias para acceder al mundo del trabajo. Otra de las vías refiere a replantear y revisar los modos en que la transmisión universitaria se está efectuando para generar miradas y acciones creativas que respondan a nuevas problemáticas. Siglo XXI, ¿se puede seguir formando profesionales de la misma manera que en el siglo $\mathrm{XX}$ ? Si la respuesta fuera afirmativa, se partiría de la suposición de que los cambios en los modos de producir, de transcurrir del tiempo, de trabajar, de los modelos sociales y aspiracionales, son sólo una apariencia ya que, en el fondo las cosas no son muy distintas que siempre. La continuidad se sostiene como una certeza que no admite la posibilidad de plantear el tránsito dentro de un nuevo paradigma. Pensar de esta manera, implicaría el posicionamiento en una mirada que sostiene que los aspectos fundamentales son trascendentes, por lo cual, los modos que se practican para comprender la realidad y operar en ella, no difieren mucho de los que se venían implementando. En esta posición la adaptación es necesaria, lo incierto debe ser evitado y el cumplimiento de los programas centra el accionar de todos los actores, favoreciendo la eficacia de los procesos y el logro de los objetivos propuestos. Las acciones universitarias son claras y precisas porque el futuro se presenta de manera determinada y en este futuro los profesionales y las tareas están explícitamente establecidas. La formación necesita acomodarse, se deberán realizar los ajustes necesarios para alcanzar las metas propuestas, pero su esencia es siempre igual.

Si la respuesta es no, se debería plantear una discusión acerca de la manera en que la educación universitaria aspiraría a formar a los futuros profesionales y también, quienes serían los sujetos elegidos para estas tareas. Esta mirada no implica abandonar la idea de programas, pero requiere no quedar centrado en ella sino ampliarla en función de la idea de estrategia. Se podría señalar, siguiendo a Morin (1990) que la realidad es cambiante, que lo nuevo puede surgir, pero que, a su vez, nada está aislado, pero fundamentalmente que todos los sujetos se encuentran enfrentados al desafío de la complejidad y ello incluye la existencia de fenómenos aleatorios, que agregan incertidumbre al pensamiento. Desde esta posición los actores se han multiplicado: docentes, estudiantes, el contexto social, epocal, entre otros. Es cierto que la idea de programa funciona en muchos aspectos, pero paralelamente, se necesitan nuevas herramientas para intervenir en la realidad, y ellas a su vez, abren nuevos campos, que antes ni siquiera existían. Desde esta perspectiva la educación universitaria se encuentra en una encrucijada brumosa, formar para un futuro que no está claramente definido (no por falta de exactitud sino por imposibilidad intrínseca) y dotar a sus egresados de herramientas que les permitan navegar por esas aguas inciertas. Un verdadero desafío.

El desafío puede ser abordado desde múltiples dimensiones. En el presente desarrollo se elegirá una perspectiva centrada en algunas problemáticas: 
- Características generales del contexto epocal y características en particular desde la perspectiva de algunos procesos en Argentina. Dentro de este apartado se desarrollarán aspectos vinculados a las transformaciones tecnológicas, el lugar de las comunicaciones, el tiempo, el sujeto epocal, el consumo, algunos valores como la felicidad, el cambio, la continuidad y la discontinuidad. También se abordarán cuestiones vinculadas al trabajo, sus transformaciones y demandas.

- Características de las formaciones universitarias: el desafío de la transmisión, los cambios y las demandas sociales, universidad para todos/algunos, el aula y su transformación. Docentes y alumnos: un vínculo complejo: Alianzas y relaciones. El aula del siglo XXI.

\section{El contexto contemporáneo}

Se podría señalar que en el plano de los cambios epocales la mirada está centrada en las modificaciones vinculadas a los modos de habitar y existir que se han ido delineando a fines del siglo XX y principios del siglo XXI. La vida en general ha migrado y se ha asentado en los medios urbanos, a la vez que ha ido adquiriendo una escala global, donde es posible, gracias a los desarrollos tecnológicos, revisar una biblioteca, no importa en qué lugar geográfico del mundo esté localizada, ver y hablar con alguien en cualquier punto del globo, conocer y recorrer una ciudad de manera virtual, estudiar a distancia y muchas otras actividades que son posibles si se cuenta con una red y una computadora, sin necesidad, de que los usuarios tengan una formación especializada.

Tiempo y espacio junto con tareas y actividades han constituido formas que ha utilizado la humanidad para organizar su existencia. Hoy se podría señalar que la noción de tiempo ha ido alcanzando dimensiones desconocidas, acortándose tanto en la dimensión geográfica como en la tecnológica. Ver a alguien, encontrarse e incluso hablar no significan lo mismo en el siglo XX que en el siglo XXI. Tiempo real, tiempo virtual han ido adquiriendo nuevos sentidos. La velocidad y la rapidez se han colado en la vida cotidiana, siendo la inmediatez uno de los atributos más valorados, sin embargo y a pesar de que la vida humana y muchos de sus procesos requieren aun de otra temporalidad.

Por el otro lado, la vida concreta de los humanos se ha extendido, en relación inversa a la duración de los objetos, de la permanencia en los puestos de trabajo, la vida en una sola vivienda o ciudad, e inclusive, en los ritmos y continuidades de las relaciones afectivas. A su vez el tiempo plantea otras cuestiones, por ejemplo, qué uso se hace de él. ¿Aprovechar el tiempo es hacer cosas? ¿Producir, es hacer una cosa? ¿Quién evalúa que aquello que se realiza es lo que se espera? ¿Quién lo espera? Plantear el uso del tiempo incorpora un actor, el sujeto.

Las maneras en que dicho sujeto es conceptualizado implicarán diferentes caminos para abordarlo y ello producirá efectos variados. Para algunos autores convivimos en una sociedad orientada a la búsqueda de experiencias emocionales, que prioriza la calidad de la vida (y de la salud), sostenida a través de la comunicación, que anhela un bienestar cada vez mayor y donde la inmediatez no deja espacio para el pensamiento y la elaboración. Es la era en la que el espíritu del consumo se ha infiltrado en todos lados: las familias, la 
religión, la política, el sindicalismo, la cultura y el tiempo disponible. Es una sociedad que huye del sufrimiento. Como señala Han (2014) la sociedad actual busca constantemente igualar, para que las únicas diferencias que existan sean las consumibles. Para este autor el sujeto es cada vez más narcisista perdiendo la capacidad de reconocer al otro en su alteridad "la erosión del otro, que tiene lugar en todos los ámbitos de la vida y va unida aun excesivo narcisismo de la propia mismidad" (Han, 2014 p. 9). La libertad como búsqueda queda desdibujada bajo el imperativo paradójico de "se libre". Para este autor los sujetos se ven forzados a mirarse a sí mismos, con poco interés hacia los otros, con poca capacidad para entramarse, relacionarse ya que el acento queda fijado en que se puede obtener y en el rendimiento. Piensa al poder como una bandera que somete a los sujetos al extremo de explotarse a sí mismos. También caracteriza a la sociedad como transparente en el sentido de que, si alguien no ha sido expuesto, no podrá dar cuenta de la propia existencia. Sostiene que esta exposición es forzada y mecánica, y que de ella resulta una pérdida de la imaginación y creatividad que transforma a los sujetos en individuos en unidimensionales (sujetos con poca capacidad para reflexionar y mucha para reproducir). Desde otra mirada se podría pensar que el mundo de lo íntimo se expone en la esfera de lo público no al modo de la invasión o el descubrimiento sino como un gesto voluntario de visibilizar y exponer aquello que antes quedaba al resguardo del ámbito privado. Los bordes entre el adentro y el afuera se esfuman y el mundo del adentro se enhebra con cuerpos que se instalan en lo público. El movimiento es múltiple mostrar, pero también curiosear y consumir lo que otros hacen (Facebook, Instagram, blogs, etc.).

La transformación de la sociedad en una sociedad de la información garantiza la circulación de una enorme cantidad de datos, que si bien, podrían saciar la curiosidad y la sed de novedades, carecen de valor si no portan algún tipo de sentido. Si se piensa en términos de cantidad es posible observar que los fenómenos son de poca duración ya que fácilmente unos son rápidamente desplazados por otros. Por otro lado, la exposición también favorece la vigilancia y la manipulación (quien no ha buscado alguna información en internet y rápidamente han aparecido a la derecha, arriba o debajo de la pantalla publicidades e información relacionada con dicha búsqueda).

La civilización consumista se distingue por el lugar central que ocupan los deseos de bienestar y la búsqueda de una vida mejor para uno mismo y los suyos.

La relación entre el tiempo ocupado por el trabajo y el ocio se ha modificado. El tiempo y el dinero destinado al ocio se han incrementado: fiestas, juegos, placeres invaden la vida cotidiana. Los modos de subsistencia se han centrado en el capitalismo, cuyo despliegue es a escala global. El capitalismo se encuentra en una nueva fase del consumo, se ha iniciado el capitalismo del hiperconsumo. Se ha pasado de una economía orientada hacia oferta a una economía orientada hacia la demanda. "La empresa orientada al producto por la empresa orientada al mercado y al consumidor" (Lipovetsky, 2007, p. 8)

Paralelamente si bien las sociedades son cada vez más ricas, y la esperanza de vida se ha incrementado (al igual que la calidad de vida en términos generales), el número de personas que viven en la precariedad y que su trabajo es insuficiente, crecen sin detención.

En La Felicidad Paradójica, Lipovetsky (2007) describe que la dinámica expansiva de las necesidades prosigue, pero cargada de nuevos sentidos tanto colectivos como individuales. La primera fase del fordismo democratizó la adquisición de bienes duraderos, la fase 
dos puso al alcance de casi todos los productos emblemáticos de la sociedad de la abundancia (televisión, autos, multiplicidad de electrodomésticos). En esta etapa por primera vez las masas accedieron a un modo de vida antes asociado a las clases privilegiadas. La seducción reemplaza la coerción, el hedonismo al deber, el gasto al ahorro y el humor a la solemnidad. Es una sociedad que alimenta la "caducidad controlada". Bajo un diluvio de signos de ligereza, frivolidad y hedonismos, la fase II se ha esforzado por deslegitimar las normas victorianas, los ideales de sacrificio y los imperativos rigoristas en beneficio de los goces privados. Se ha pasado de la mirada en el futuro hacia el presente, la satisfacción inmediata, Es la caída de la modernidad disciplinaria. La sociedad del posconsumo es una ruptura dentro de la continuidad. El sistema posfordiano se presenta con profundas alteraciones en la forma de estimular la venta, en los comportamientos y los imaginarios del consumo. Las industrias y servicios buscan estimular las necesidades y los signos que se asocian a modos de vida valorados a través de estrategias de personalización que permiten segmentar, diferenciar, e incentivar la búsqueda de lo más nuevo, lo último. Como señala Lipovetsky (2007) es la sociedad del hiperconsumo.

Además, se puede sumar los efectos de la digitalización de la vida cotidiana. Como señalan Schiavo y Gelfuso (2018), en nuestro país la tecnología digital implicó, a partir de la última década del siglo pasado, la incorporación de una nueva dimensión espacio temporal que permitió comenzar a deambular y circular por un nuevo territorio, el virtual, que gradualmente se fue imponiendo en la mayoría de las actividades humanas. Espacio inédito y fruto de la creación humana. Como señalan estos autores el escenario urbano es característico del siglo XXI, pero la idea de estas ciudades es constituirse y trascender como ciudades inteligentes que viabilicen diferentes maneras de habitar el entorno urbano ya sea como un espacio presencial o virtual, local y global.

El mundo actual es fundamentalmente urbano. Se caracterizan por constituirse en lugares muy dinámicos culturalmente, con mayor desarrollo de la capacidad cultural y donde tienen amplias potencialidades las innovaciones. Puede observarse que en general los innovadores eligen vivir en entornos sociales con alta calidad de vida, con un nivel económico también elevado, dando lugar a lo que Florida (citado en Finquelievich, 2018, p. 228) llama "la clase creativa" que a su vez llama a otros creativos. Ello a su vez atrae a empresas y capital. Varios países también han observado este fenómeno y han posibilitado y facilitado que ciudades medianas y pequeñas, favorezcan la incorporación de emprendedores, no sin la actuación y participación de las universidades a través del desarrollo de carreras, redes de conocimientos, interacción también con el mundo científico, creación de polos tecnológicos, facilidades fiscales, buena calidad ambiental, además de un entorno más amigable. También las actividades principales de los estados han sufrido modificaciones. En términos generales se observa que la producción ha ido perdiendo su lugar central pasando a tenerlo la prestación de servicios que además se encuentra relacionada con la aspiración de las ciudades a convertirse en "inteligentes".

Nuevos escenarios requieren replanteos estratégicos y en ellos la formación profesional no puede quedar afuera. La educación universitaria necesita revisar sus contenidos, poner en cuestión sus vínculos hacia adentro y hacia fuera de la institución y también examinar los modos en que se implementa y transforma su ejercicio. Una de las cuestiones más complejas refiere a que sus tiempos son más lentos que los cambios tecnológicos e incluso se plan- 
tea que su aggiornamiento facilitaría la aparición de nuevos puestos de trabajo El mundo laboral también se encuentra en plena transformación, y coincidiendo con Finquelievich (2018), ya no es la amenaza de la revolución industrial la que echará por tierra puestos laborales sino la tecnificación digital la que inclinará la balanza para la desaparición de algunos puestos y la creación de nuevos nichos para otros.

En relación con el mundo del trabajo, muchos autores sostienen que los trabajos que requieren habilidades medias no desaparecerán, sólo implican una combinación entre habilidades sociales y cierta adaptabilidad. Mientras que otros, los nuevos artesanos, entre ellos los técnicos de las nuevas tecnologías tendrán su lugar.

La informatización no reduce la cantidad de empleos pero existe una tendencia a degradar la calidad de los empleos para muchos trabajadores. Los trabajadores que no tienen una educación técnica o universitaria tienen una remuneración baja, son numerosos puestos y se encuentran clasificados en torno a lo manual. En este sentido las cifras que correlacionan empleo y formación en la Argentina señalan que los que tienen menor formación poseen una tasa de desempleo menor que los que tienen títulos de grado, si ello es cruzado por clase social y sexo. En este sentido Tiramonti (2019) señala:

Lo que ocultan las estadísticas agregadas y develan aquellas que se desagregan por nivel socioeconómico es que, en nuestro país, lo que define la empleabilidad de las personas no es su titulación, sino su origen social. La carrera abierta al talento, que según Hobsbawm posibilitó las revoluciones burguesas, en la Argentina no se da. La suerte laboral de las personas está más atada a su capital social (amistades, contactos, modos de presentarse, hábitos lingüísticos y de relacionarse con los otros) que con las acreditaciones educativas que posee.

Sin embargo, se podría pensar, que no es sin la formación universitaria, que el "capital social" potencia las posibilidades laborales del futuro profesional.

\section{El trabajo}

El trabajo en la era moderna se constituye en uno de los símbolos que cualifican la pertenencia a una ciudadanía social. Desde esta mirada, tener un puesto en el mercado laboral viabiliza un camino para formar parte del mundo, donde formar parte es participar a través de acciones. Es a través de la participación (léase acciones) que se suscribe la garantía de pertenencia. Esta pertenencia se ha ido modificando junto con la transformación de los formatos laborales que a lo largo del siglo XX y comienzo del XXI fueron pasando del modelo de contratos fijos hacia modelos más flexibles asociados a la desregulación, bordeando a la vez, en algunos sectores, la denominada precarización laboral.

El cambio de formato laboral puede también ser observado hoy en la respuesta que los jóvenes dan acerca de su percepción en cuanto al futuro laboral, la mayoría no se imagina en un trabajo para toda la vida. En estos jóvenes el cambio atestigua una puesta en valor en el camino hacia el progreso en la carrera profesional. En este sentido, la fluidez, al decir de 
Bauman teñiría de manera definitiva las formas en las que se van construyendo los imaginarios con relación al mundo del trabajo. Sin embargo y junto a estas modificaciones aspiracionales, el empleo sigue siendo uno de los elementos creadores de diferencias sociales (Alonso y Fernandez Rodriguez, 2018). Valor que ha portado el trabajo desde la era de la Ilustración, diferenciándose claramente de la época de los griegos donde el trabajo y las actividades manuales eran poco estimadas ya que no sólo mantenían al hombre pegado a la materia sino fundamentalmente porque iban en detrimento de su autonomía al someterlo a las necesidades de la vida y por sobre todo al dominio del amo por sobre el esclavo. El trabajo en la era posfordista, siguiendo a Virno (Virno, 2016) se ha transformado pasando de la silenciosa maquinaria organizada y ordenada de manera específica a un modelo donde la producción necesita de la interacción entre los sujetos y además de sus competencias específicas tanto cognitivas como lingüísticas por ello señala que “...es lícito sostener que una parte significativa de los así llamados medios de producción consiste en técnicas y procedimientos comunicativos" (Virno, p. 61).

Es interesante describir los atributos que los trabajadores deben poseer según este autor:

Que estén habituados a moverse de un lado a otro, que sean capaces de acomodarse a las más bruscas reconversiones, que sean adaptables para cambiar de una dependencia a otras, que sean dúctiles para cambiar las reglas de juego, que sepan llevar adelante interacciones lingüísticas banales, que demuestren destreza para elegir y sepan manejar diversas alternativas (Virno, p. 85).

Virno (2016) señala que estos requisitos no son resultado del disciplinamiento industrial y ello se podría parafrasear señalando que tampoco es fruto del proceso educativo (en términos modernos), que a grandes rasgos justamente intenta disciplinar y combatir (e incluso eliminar) aquellos elementos que son relacionados con lo que irrumpe y dificulta la cumplimentación de la programación, con aquello que favorece la creatividad, ya que ella se encuentra por fuera del control del docente y por las serias dificultades que plantea todo aquello que quede por fuera de las reglas y la ley. En este sentido el armado de una trama entre las singularidades y lo universal, se constituye en un punto complejo que se juega en el ejercicio concreto del encuentro entre docentes y alumnos. Entonces si ello no se adquiere en el ámbito educativo, se adquiere en otro lado. Este autor señala que es la socialización que se produce por fuera del ámbito institucional. Esa habilidad de no tener hábitos duraderos son las herramientas que luego la empresa usufructuará en su provecho. Se podría decir que la fuerza de trabajo es entendida como una potencia para producir. Esta potencia estaría definida por la totalidad de las aptitudes físicas e intelectuales que residen en la corporalidad. ¿Podrá la educación universitaria favorecer la potencia y singularidad de sus futuros profesionales sin atar su destino al peso de modos tradicionales de pensamiento y producción? 


\section{Universidad y docencia}

Pensar la universidad, implica ubicar un tiempo y un espacio particular ya que sin estas coordenadas se imposibilita una reflexión que intentan deconstruir para comprender las marcas de cada época. Las universidades han sido tradicionalmente espacios destinados a la formación de profesionales (docencia), producción de nuevos conocimientos (investigación) y prácticas en el campo de lo social (área de extensión).

La enseñanza superior en la República Argentina tiene su origen en la provincia de Córdoba, donde los jesuitas fundaron el Colegio Máximo. Esta institución fue organizada como los claustros medievales y se dedicó por casi un siglo exclusivamente a la enseñanza de la teología. En el siglo XVIII, tras la expulsión de los jesuitas, se incorpora la enseñanza del derecho. En 1821 se crea la universidad de Buenos Aires (UBA) con una organización más moderna, centrada en las necesidades de su entorno y con la misión de resolver las problemáticas de los habitantes de la ciudad. Por ello las primeras áreas fueron: Medicina y Ciencias Exactas, Primeras Letras, Jurisprudencia y Ciencias Sagradas. Ambas universidades tuvieron durante estos siglos un papel preponderante en la vida política e institucional ya que formaron a muchos de los dirigentes que tuvieron importantes cargos tanto en lo estrictamente político como así también en el área burocrática. Con los cambios políticos institucionales de finales de siglo XIX, la universidad queda definida como aquella que se dedica a certificar el ejercicio de la labor profesional: médicos, abogados, ingenieros, entre otros. De esta manera la universidad funcionó como un engranaje clave, que facilitó el ascenso social, a una sociedad marcada por las migraciones, las promesas de cambio, la movilidad y el progreso. Durante el siglo XX se amplió el espectro de conocimientos y profesiones, a la vez que se sostuvo la gratuidad y el ingreso irrestricto (con algunas excepciones). En la segunda mitad del siglo se crean las primeras universidad privadas, $y$ a fines del siglo XX se incrementa el número de universidades públicas. La masividad es una de las características de la universidad argentina, pero lo es también su alta tasa de deserción. En este escenario de crecimiento y masividad la formación del profesional se ha transformado en una formación continua y la investigación no sólo en la producción del conocimiento para sí, sino también al servicio de la producción y comercialización del conocimiento en el campo social. En este proceso la investigación constituía una de las maneras de vincularse con el entorno y el área de extensión se desarrollaba desde una mirada asistencialista. En el campo de la extensión también se han producido cambios a través de la intervención e incorporación de la investigación y la docencia transformando su función primigenia en un accionar destinado a favorecer la intervención y proyección social de las universidades. El mundo socio-cultural en sentido amplio y el proyecto educativo en particular imprimen sus marcas y demandas a la educación universitaria y ésta a su vez, como fruto de la interacción se constituye en una representante de relevancia en el campo de la producción social. En el caso particular de Argentina no es posible desconocer que el estado se ha retirado de muchas áreas de intervención comunitaria, abandonando su accionar. Es desde este espacio que se habilitaron nuevos puntos en las agendas académicas de las universidades, dando lugar a relevamientos sociales, políticos, económicos como así también la gestión y generación de movimientos culturales y artísticos que facilitaron el pasaje de las llamadas "nuevas universidades" a constituirse en actores políticos de peso. 
Entonces la historia permite señalar que el área profesional y la formación de los profesionales ha sido uno de los elementos que troncalmente le han dado entidad a las casas de estudio. El desarrollo de áreas de investigación junto con la resolución de problemas y nuevos conocimientos han conformado un entramado cuyo peso ha sido significativo para la existencia de los estados modernos. Por ello, el mundo laboral y sus modificaciones y demandas se encuentran intrínsecamente relacionadas con la calidad y especificidad de la educación universitaria.

La idea del cambio, de las transformaciones y los modos en que están se implementan en los proyectos nacionales no es ingenua. Ninguna academia puede tener su lugar si no se encuentra relacionada con el contexto donde desarrolla sus actividades ya que es un intercambio del que todos se nutren y porque su existencia, como ya se señaló anteriormente está condicionada por la misma interacción en el campo social.

Hablar de cambio y transformaciones plantea la necesitad de nuevos modos de interrogar y nuevas formas de responder. Salir de lo habitual para habilitar lo incierto.

Puget (2014) sostiene que la continuidad trabaja con lo esperado pero la discontinuidad incorpora la espera de lo esperado, pero también de lo inesperado. En este sentido la construcción de la experiencia no tiene pasado, pero puede crearlo. Presente y pasado conviven y podría señalarse que una de las cuestiones que dificultan el trabajo con las situaciones problemáticas se encuentra asociado a los modos en los que se habitan esas mismas situaciones. Novedades y diversidad de puntos de vista complejizan la posición de los sujetos en el mundo y a veces se corre el riesgo paradojal de que lo nuevo quede desestimado bajo la mirada de lo viejo y que a su vez lo nuevo reemplace lo viejo. Quizás no es sesgar hacia uno u otro lado sino complejizar y hacer lugar a las diferencias.

Históricamente la preocupación por la calidad de la enseñanza universitaria empieza a tomar cuerpo en nuestro país en la última década del siglo XX, donde comienzan a implementarse procesos de evaluación institucional que junto con el incremento de la matrícula requirió que los gobiernos acompañasen esa expansión con políticas que controlasen la calidad universitaria a través de principalmente dos acciones: por un lado, la creación de la agencia específica de control, para la evaluación y acreditación de universidades Consejo Nacional de Evaluación y Acreditación Universitaria (CONEAU) y por el otro, una serie de acciones que orientan de manera indirecta que se espera de las casas de estudio (por ejemplo con subsidios económicos).

La calidad lleva a interrogar sobre que profesionales se necesitan, que conocimientos son relevantes y cuál es el rol de las universidades en este proceso. Uno de los aspectos a considerar, como señala Fernandez Lamarra (2019), es que la universidad como institución paradigmática de producción y transferencia de conocimientos científicos, profesionales y artísticos, debería también constituirse en un agente de innovación. En este sentido, y de acuerdo a las exigencias de la época y a la rapidez con que se demandan respuestas, las universidades deberían privilegiar su lugar en relación a las acciones innovadoras por sobre su tradicional desempeño, evitando que estas acciones afecten la acreditación que las carreras requieren (y que la CONEAU certifica) y la existencia de la institución misma. Hoy la formación del profesional se ha transformado en una formación continua y la investigación no sólo en la producción del conocimiento para sí sino también al servicio de la producción y comercialización del conocimiento en el campo social. En este proceso 
la investigación constituía una de las maneras de vincularse con el entorno y el área de extensión se desarrollaba desde una mirada asistencialista. En el campo de la extensión también se han producido cambios a través de la intervención e incorporación de la investigación y la docencia transformando su función primigenia en un accionar que favorece la intervención y proyección social de las universidades.

Estos aspectos no están aislados del proyecto educativo en general y de los modos en que las universidades se van insertando en el campo social, estimulando el replanteao de los modos en los que se construye la mirada del futuro profesional y los planes para su formación. En este sentido salir de la idea de isla del conocimiento (universidad aislada propio del paradigma de la modernidad) implica pasar de un modelo que trabaja con la determinación y la disyunción, a un modelo que no busca aislar para entender y conocer, sino que es desde su pertenencia a la red que es posible comprender, intervenir y/o crear.

Un aspecto que reclama cierta reflexión en relación con el campo social refiere a las demandas que la misma sociedad (a través de sus distintos organismos de control) plantea. Se espera que la educación construya sujetos habilitados a ejercer profesionalmente y que se comporten como ciudadanos pero ya no de acuerdo a los términos y necesidades del estado moderno de los siglos pasados (con el fin de alimentar y sostener la maquinaria del estado moderno) sino que también busca que las acciones institucionales educativas reparen uno de los aspectos que pone en peligro la existencia misma del estado: la desigualdad social (sin que resulte claro en qué términos es pensado el mismo). Quizás aquí habría que preguntar quién garantiza el funcionamiento social si el estado esta caído y debilitado, en otros términos, si cae el supuesto que organizaba la actividad subjetiva de acuerdo con una ley trascendente. En este sentido, las quejas de unos y otros, las expectativas diferentes y frustrantes podrían ser pensadas como síntomas de una resistencia que no hace más que marcar la defensa de un pasado que reniega del tiempo actual. Es interesante considerar, como señalaba Lewkowicz (Lewcowicz, 2001), que la resistencia pareciera querer conservar una hegemonía pasada donde resistir estaría más relacionado con el sostenimiento de modalidades obsoletas afines al servicio de obstruir y dificultar el encuentro con un presente donde el devenir y la transformación se imponen.

\section{El maestro y la transmisión. El aula}

Pensar en la institución universitaria implica revisar el lugar de la transmisión y quienes están a cargo de la misma. Tradicionalmente como señala Ranciére (2007 p. 17)

El acto esencial del maestro era explicar, despejar los elementos simples del conocimiento y hacer que su simplicidad de principio concuerde con la simplicidad de hecho que caracteriza a los espíritus jóvenes e ignorantes. Enseñar era, al mismo tiempo, transmitir conocimientos formar espíritus, conduciéndolos, según una progresión ordenada, de los más simples a los más complejos. 
Este modelo, llamado el “orden explicador” se operativiza a través de una explicación oral para poder explicar la explicación escrita y así ubicar a su interlocutor como alguien que no posee una capacidad propia para comprender. Este modelo, denominado por Ranciére como el principio del embrutecimiento, necesita de alguien en la posesión del saber, que intentará por todos los medios, transmitir conocimientos para que el otro, el alumno pueda comprender y aprender, que sólo de la mano de su maestro comprenderá que si no le explican no comprende.

Quien quiere conciliar orden y progreso encuentra con toda naturalidad su modelo en una institución que simboliza su unión: la institución pedagógica, el lugar -material y simbólico- donde el ejercicio de la autoridad y la sumisión de los sujetos no tiene otro objetivo que el de la progresión de esos sujetos hasta el alcanzar límites de su capacidad: el conocimiento de las materias del programa para la mayoría, la capacidad de convertirse, llegado el momento, en maestros, para los mejores (Ranciére, 2007 p. 8).

La revolución en las comunicaciones ha perforado el lugar central del maestro en tanto fuente privilegiada del conocimiento, se puede aprender en otros espacios, sin obedecer a un tiempo y un formato diseñado para tal fin. El acceso al conocimiento se ha vuelto polifónico y ello permite cuestionar y revisar el concepto de transmisión. El profesor no es el único que llama la atención del alumno, y debe competir con la multiplicidad de estímulos a los que la vida urbana tiene a sus habitantes acostumbrados. ¿Es que el profesor se ha quedado sin lugar, sin un quehacer específico? Si se acepta que ha habido cambios, los modos en los sujetos se relacionan con los diferentes objetos también se han modificado (pues éstos ya no son los mismos sujetos ni tampoco son los mismos objetos). En este sentido, las preguntas acerca del lugar del maestro revelan que la diferencia entre el ejercicio de la docencia, las demandas y lo esperado en el campo de la formación se encuentran en tensión y requieren una impostergable revisión. Qué espera el docente de su trabajo y cuáles son las condiciones en las que es posible su transformación, no son temáticas simples y además están sujetas a la singularidad de cada institución. Qué esperan los alumnos de su tránsito por la universidad genera nuevos interrogantes que a su vez se complejizan con las demandas y expectativas de todos los actores sociales que conforman una comunidad en particular.

\section{El aula y sus bordes}

La diversidad, como aspiración, sería otra de las marcas que caracterizan la formación universitaria (siempre sujeta a las normas de acreditación), pero el aula, como espacio de transmisión y producción se convierte en un vínculo que no sólo reúne a docentes y alumnos, sino que también conecta y bordea la generación de una dimensión que es transversal a la singularidad de cada casa de estudio. En sentido amplio puede señalarse que el aula (ya sea presencial y/o virtual) es un espacio en el que se produce el encuentro entre 
sujetos que portan diferencias y en el que se producen efectos de alteridad. Entre estos participantes se producen reconocimientos mutuos (devenidos de la misma mutualidad) y aunque se encuentran en posiciones asimétricas (en términos de responsabilidades institucionales y éticas), todos portan conocimientos, pueden intercambiar experiencias e ideas, colaborar, enseñar, debatir, aprender, pero fundamentalmente habilitar y explorar las herramientas para producir nuevos saberes.

El aula como lugar, con sus marcas y su carga simbólica es el espacio privilegiado para este tipo de encuentros, pero también para revisar y reflexionar acerca de las tensiones que en de manera invisible sostienen y constituyen este particular entramado que permite y vehiculiza los intercambios. Es la noción de poder, entendida como el fluir de fuerzas en tensión y continuo movimiento, la que dimensiona la trama. No es el poder de tener sino el poder de hacer. Es un poder pensado como potencia, la potencia de hacer con otros, que sólo el devenir de los encuentros permitirá desarrollar y delinear en cuanto a sus posibilidades. En este sentido se constituye en un espacio especial, en tanto catalizador y favorecedor del juego de las diferencias, el despertar de los conflictos que junto con la riqueza de los intercambios podrían propiciar el surgimiento de nuevos sentidos. El riesgo es la invisibilización (en incluso negación) de las diferencias (entendidas como aquello que difiere) o peor aún, de los otros como sujetos en función de ideas preconcebidas o de modelos que adormecen las potencialidades y el devenir de los encuentros (favoreciendo posiciones extremas, miradas únicas y/o verdades absolutas).

La idea de poder se relaciona con saber (aquellos que tienen saber tienen poder y viceversa). Si bien poder y saber se relacionan e implican, el saber aquí es pensado como un saber que no busca avasallar, impresionar ni empobrecer, sino un saber que se origina en el lazo con el/los otro/os, una posibilidad que sólo aparece en y a partir del encuentro. Un saber a transmitir, a donar, que no es el único, sino un saber entre otros, que puede ser transmitidos, favoreciendo la continuidad cultural (ello entendido como el magma que genera diferentes producciones cuyas formas no son fijas ni certeras). Podría plantearse que ello bordearía el poder de la autoridad, pensando a la misma en relación con la idea de "estar autorizado" para hacer algo. Ya no se referiría al lugar del docente frente a los alumnos sino al de cada uno de los sujetos que participan de esa trama. Es la alianza entre sus participantes (aunque este fuera algo temporal) la que visibiliza a través de sus efectos, las marcas que el participar de ese proceso dejan en sus subjetividades. Marcas que no podrían haber sido posibles sin el trabajo con y desde ese vínculo. En esta dimensión el aula es la vía regia favorecedora de experiencias de aprendizaje, que se diferencian de otras dado que su especificidad es valorada socialmente, ya que por su misma existencia habilita y acredita a los sujetos y a la comunidad en general, la posesión de cierta formación y saber.

Hablar del saber implica también incluir saberes de tipo autoritarios. Este tipo de saberes estarían más del lado del saber cerrado, del saber que se impone, que no sirve para el intercambio, que cierra los circuitos de producción y que se sostiene sólo a través del uso de la fuerza, la amenaza y la imposición.

Diferenciar poder, autoridad y autoritarismo, lleva también a desmarcar saber y poder, para pensar en "saber hacer con el otro". Hacer con otros, construir y descubrir saberes junto a otros remite nuevamente el espacio áulico como un lugar posible para el encuentro, la producción y la creación. Transmitir refiere a las ideas de trasladar, transferir, ceder. 
Ello implica que algo atraviesa a todos los partícipes de ese encuentro. Encuentro conlleva la idea de producción de algo nuevo. Ese algo nuevo ¿se puede pensar como un "producir con"? ¿Se puede pensar como un "aprender con"?

El aula como construcción imaginaria porta ciertos supuestos y en entre ellos es habitual encontrar aquellos que traen los alumnos y aquellos que sostiene los docentes. En el plano de los alumnos es frecuente la idea de que "se viene a recibir", recibir algo que no se tenía, que no se sabe si se quiere, pero está claro que cualquiera sea la motivación implicará algunos afectos: curiosidad, temor, amenaza, interés y hasta excitación y hastío. Pero indefectiblemente implicará algún tipo de movimiento para incorporarlo, apartarlo, modificarlo, usarlo, destruirlo, transformarlo y e incluso olvidarlo. Por otro lado, los docentes no encuentran a los alumnos para los cuales fueron formados ni tampoco resultan dóciles e interesados en el aprendizaje lineal y en muchos de los contenidos que las currículas proponen. El cruce de expectativas en un escenario en el cual interactúan políticas educativas diferentes, ritmos cotidianos transformados por la instantaneidad de la cronología actual y la cotidianeidad atravesada por eventos y pantallas, que junto con las promesas de satisfacción a medida e inmediata, chocan con modelos de aprendizaje cuyos procesos requieren otros tiempos y donde sus resultados ya no certifican ni aseguran, de manera certera, un lugar en el mundo (entiéndase como se señaló en la primera parte, un lugar en el mundo del trabajo y del consumo, una existencia visible y pública). El aula impone un trabajo en tiempo presente, pero su horizonte es el futuro.

El sujeto actual necesita otras competencias, competencias que no se agotan en le extensión y/o profundización de los contenidos. Competencias que permitan potenciar y viabilizar otros modos de habitar lo contemporáneo y que, a la vez, transformen algo del malestar en una accionar productivo y creativo. Es el aula como espacio simbólico, una construcción que a través de su misma deconstrucción puede producir los andamiajes para el trabajo con lo novedoso y la construcción de nuevos significados.

Valorar la curiosidad, la iniciativa, la consideración y atención hacia el semejante, la construcción de reglas inmanentes, entre otros aspectos, podrían convertirse en herramientas al servicio de fomentar la multiplicidad, de salir de los determinismos y las etiquetas. No es el cambio en el espacio físico ni tampoco sólo el cambio tecnológico, es la co-contrucción de un espacio favorecedor de los intercambios y donde la alianza entre pares (que no quiere decir entre iguales) viabiliza el trabajo con lo que hasta ese encuentro era desconocido o incluso no tenía existencia y habilita la posibilidad de producción de nuevos sentidos. Puget (2015, p. 48) plantea que "el pensar entre dos o más genera un hacer algo a partir de lo que la alteridad de cada uno impone y crea. El motor es lo ajeno y la alteridad, y ello siempre excede". Este punto de vista profundiza la noción del "entre" ya que lo producido no pertenece a ninguno en particular sino a todos. Pensar entre otros permite escenificar que la riqueza del encuentro es la imposibilidad de sostener el privilegio de un único punto de vista, de la puesta en acción de la construcción y la deconstrucción de las ideas, y que los otros pueden conmover las posiciones más certeras.

Compartir con otros también puede ser asociado a entender, aquella posición donde un sujeto se ubica frente a su decir invitando a los otros a participar de esa experiencia. En este sentido el "vínculo par" (todos los que conforman y habitan el aula en un momento determinado) habilita a experienciar "la insuficiencia de los distintos puntos de vista" 
como señalan Mauer et al. (2014, p. 197) y a la vez producir y crear con otros, nuevas maneras de habitar el "espacio aúlico". Éste es posible en tanto los participantes se muestren confiados, responsables de sus producciones y no sustenten sus posiciones en una obediencia a un saber y una realidad por fuera de sí mismos, camino que incluye el disenso y el desencuentro.

En este sentido y siguiendo nuevamente a Ranciére, puede señalarse que "maestro es quien mantiene a quien busca en su camino, en donde él es el único que busca y no deja de buscar" (2007 p. 51). Entendiéndose el camino como un trazado incierto que se va configurando por el juego de la curiosidad, el interés, la potencia de habilitar y construir nuevas miradas sobre el mundo. Conocer y despertar la curiosidad se entraman ya que en el mismo suceder algo de lo anterior se pierde, es necesario abandonar el estado de certeza, posibilitar una zona enigmática y entonces, puede ser posible que el asombro aparezca.

Ranciére (2007) se interesa por cuestionar filosóficamente la posición pedagógica, en este aspecto desde un modelo binario, señala que la idea de instruir puede tener efectos absolutamente opuestos: reducir la capacidad de aprender o forzar esa capacidad para que se desarrolle en todas sus posibilidades. A este último acto lo denomina emancipación. Podría señalarse que no es posible pensar en la emancipación sin la presencia del pensamiento. Puget (2015) propone la existencia de un pensar necesario que tiene un carácter utilitario y también de un pensar creativo, caracterizado por una imaginación sin límites, que no tiene un origen preciso y que es fruto del juego de infinitas variables un "hacer desde la indeterminación, destituyendo certezas" (2015 p. 50).

Es en el aula, y entre sus habitantes que se habilita una dimensión donde es posible constituir una amalgama entre saberes y conocimientos que pueden estar cristalizados. Pero éstos también pueden ser conceptualizados como conjuntos que fluyen y cuya producción es generadora de experiencias que despiertan asombros, compromisos con la vida y los semejantes y la posibilidad de habilitar la curiosidad como una llave que abre las puertas a una zona enigmática que despierta el placer por conocer y descubrir lo nuevo.

El aula hoy no está llena de promesas sino que es un lugar en permanente construcción, que no tiene claramente sus bordes definidos por las paredes instituciones sino que es una especie de puente, de borde que habilita a deconstruir algunos de los rasgos de lo contemporáneo: frente a una mirada que posiciona el éxito en lo individual, en la invisibilidad de las prácticas colectivas, en la aceleración del tiempo y en la evanescencia de los contactos se apuesta a pensar, a debatir, a discutir, a desechar, a enfrentar, a exponer pero fundamentalmente a sostener un hacer con los otros que abre la posibilidad a un nuevos inicios, donde la curiosidad y el misterio de lo desconocido tienen su protagonismo y donde es el entramado que en su hacer colectivo produce y genera el devenir de nuevas creaciones.

\section{Referencias Bibliográficas}

Alonso, L. y Fernandez Rodriguez, C. (2009). El trabajo en la era posfordista: un malestar permanente. En revista PAPELES de relaciones ecosociales y cambio global N 108 pp 
21-33. Disponible en www.fuhem.es/.../El_trabajo_en_la_era_posfordista_L.E.ALONS O_C.J.FERNANDEZ

Buchbinder, P. Las Universidades en la Argentina: una brevísima historia. Disponible en http://www.vocesenelfenix.com/content/las-universidades-en-la-argentina-unabrev\%C3\%ADsima-historia.

Fernandez Lamarra, N. Repensando la calidad de la educación superior: el contexto, las definiciones y los desafíos pendientes. Disponible en http://www.vocesenelfenix.com/ content/repensando-la-calidad-de-la-educaci $\% \mathrm{C} 3 \% \mathrm{~B} 3 \mathrm{n}$-superior-el-contexto-lasdefiniciones-y-los-desaf\%C3\%ADos-p (2019)

Finquelievich, S. (2018): Consideraciones para analizar las ciudades en la cuarta revolución industrial. En S. Martinez Lago (comp), Acerca de la apropiación de tecnologías: teorías, estudios y debates. Rada Tilly: Del Gato Gris; Ciudad Autónoma de Buenos Aires: IIGG; Ciudad Autónoma de Buenos Aires: Red de Investigadores sobre Apropiación de Tecnologías.

Han, B. (2014). La agonía del Eros. Buenos Aires: Herder Editorial.

Lewkowicz (2001). Pensar sin estado. La subjetividad en la era de la fluidez. Buenos Aires: Paidos.

Lipovetsky, G. (2007). La Felicidad Paradójica. Barcelona: Anagrama.

Mauer, S.; Moscona S. y Resnizky, S. (2014). Dispositivos clínicos en Psicoanálisis. Buenos Aires: Letra Viva librería y editorial.

Morin, E. (1990). Introducción al Pensamiento Complejo. Barcelona: Gedisa.

Puget, J. (2015). Subjetivación Discontinua y Psicoanálisis. Incetidumbres y certezas. Buenos Aires: Lugar Editorial.

Ranciére, J. (2007). El Maestro ignorante. Cinco lecciones sobre la emancipación intelectual. Buenos Aires: Libros del Zorzal.

Schiavo, E. y Gelfuso, A. (2018). Apropiación de tecnologías digitales en la ciudad inteligente. En S. Martinez Lago (comp), Acerca de la apropiación de tecnologías: teorías, estudios y debates. Rada Tilly: Del Gato Gris; Ciudad Autónoma de Buenos Aires: IIGG; Ciudad Autónoma de Buenos Aires: Red de Investigadores sobre Apropiación de Tecnologías.

Virno, P. (2016). Gramática de la Multitud. Para un análisis de las formas de vida contemporáneas. Madrid: Traficantes de sueños.

Tiramonti. G. (2019). El diploma no garantiza un empleo. Disponible en https://www. lanacion.com.ar/opinion/el-diploma-no-garantiza-un-empleo-nid2212731

\section{Bibliografía}

Bauman, Z. (2006). Amor líquido. Acerca de la fragilidad de los vínculos humanos. Buenos Aires: Fondo de Cultura Económica.

Bauman, Z. (2007). Vida de consumo. Buenos Aires: Fondo de Cultura Económica.

Belmes, D. (2012). "Reflexiones desde el aula” en Reflexión Académica en Diseño y Comunicación No XIX (Año XIII, Vol. 19, Agosto 2012, Buenos Aires, Argentina).

Belmes, D. (2018). Estudiar hoy: ¿es otra forma? En Reflexion Académica en Diseño y Comunicación $\mathrm{N}^{\circ}$ XXXV (Año XIX, Vol ${ }^{\circ} 35$, Agosto 2018, Buenos Aires, Argentina). 
Belmes, D. (2018). Indicadores Aspiracionales, ¿ cómo pensar logros en épocas de fluidez? En Reflexión Académica en Diseño y Comunicación $\mathrm{N}^{\circ}$ XXXV (Año XIX, Vol ${ }^{\circ} 35$, Agosto 2018, Buenos Aires, Argentina).

Cabello, R. (2018). 20 minutos en el futuro. Distancias y relaciones interpersonales en el espacio digital cambiante. Buenos Aires: Prometeo Libros.

Díaz, E. (2010). Las grietas del control. Vida, vigilancia y caos. Buenos Aires: Editorial Biblios. Mendez, M. (2014). Procesos de Subjetivación. Ensayos entres Antropología y Educación. Paraná: Fundación La Hendija

Ranciere, J. (2012). El desacuerdo. Política y Filosofía. Buenos Aires: Ediciones Nueva Visión. Sibilia, P. (2008). La intimidad como espectáculo. Buenos Aires: Fondo de Cultura Económica. Steiner, G. (2011). Lecciones de los maestros. Buenos Aires: Debolsillo.

\begin{abstract}
The aim of this paper is to share some reflections on the role of university education in connection with the socio-cultural context and the world of work. The 21st century presents a changing and convulsed scenario

due to the new forms of capitalism, the changes in communications, the advances of technology in everyday life, the growing centrality of the urban territory, changes in consumption, and reconversions of all types in the world that have transformed the requirements and skills for jobs. The question about training is not naive. Education in Argentina has historically held a privileged position and, also has raised a series of debates and tensions. The current situation raises the question about the new challenges not only in terms of its contents but also in the ways in which its practices are implemented. This paper is focused on the following questions: what needs to be taught?, what can be thought about the transmission of knowledge?, who are the teachers and who are the students?, what are the proposals about the transformation of the classroom as a meeting space and what is the relationship with the future?
\end{abstract}

Keywords: university education - jobs skills - transmission of knowledge - classroom faculty - students.

Resumo: A proposta deste trabalho é compartilhar algumas reflexões sobre o lugar que o conhecimento universitário ocupa em relação ao contexto sociocultural e ao mundo do trabalho. O século XXI é apresentado como um cenário em mudança e convulsionado pelas novas formas de capitalismo, as mudanças nas comunicações, a tecnologização da vida cotidiana, a crescente centralização do urbano, as mudanças no consumo e as reconversões no mundo, trabalho que transformou os requisitos para empregos. A questão sobre o treinamento não é ingênua. A educação na Argentina historicamente teve um lugar importante, que gerou debates e tensões. No nível universitário, o momento atual apresenta uma série de desafios, não apenas em termos de conteúdo, mas também no que se refere à maneira como suas práticas são implementadas. O que deve ser ensinado, o que pode ser pensado sobre a transmissão de conhecimento, quem são os professores e quem 
são os alunos, quais são as propostas sobre a transformação da sala de aula como local de encontro e qual é a relação com o futuro. Algumas das perguntas que norteiam este artigo.

Palavras chave: formação universitária - requisitos de trabalho - sala de aula - transmissão - professores - alunos.

[Las traducciones de los abstracts fueron supervisadas por el autor de cada artículo] 